\title{
Estudos comparativos de efeito Bohr em hemoglobinas de Osteoglossum bicirrhossum e Pterygoplicthys pardalis
}

\author{
Maria Isabel Galdames Portus (") \\ Aldo Focesi Junior ("*)
}

\begin{abstract}
Resumo
Alguns aspectos comparativos entre hemoglobinas de duas espécies de peixes do rio Amazonas foram análisadas, com a finalidade de estabelecer as bases moleculares de suas propriedades funcionais, bem como, as interações entre estas proteinas com outras mo'éculas e ions. As espécies estudadas possuem diferentes características fisiológicas de respiração, atividade motora e meio ecológico. A hemoglobina de aruanã por eletroforese em poliacrilamida apresentou um componente único com pronunciado efeito Bohr e Root. Os valores de " $n$ " na equaçăo de Hill, mostram variações apreciáveis com o $\mathrm{pH}$, tanto na presença como na ausência de ATP $\left(10^{-3} \mathrm{M}\right)$. As hemoglobinas de acari-bodó no hemolisado total não apresentaram efeito Bohr, contudo os quatro componentes isolados mostraram propriedades funcionais diferentes. $\mathrm{O}$ primeiro $\mathrm{Hbl}$, mostrou pequeno efeito Bohr reverso e uma acentuada influência de ATP $\left(10^{-3} \mathrm{M}\right)$ em $\mathrm{pH}$ baixo, sobre este efeito tornando-se normal, enquanto que os três outros componentes apresentaram efeito Bohr normal e nenhuma influência de ATP sobre ele. Nossos resultados mostram claramente a capacidade de adaptação molecular das hemoglobinas destes peixes às condições fisiológicas e ambientais a que eles estão sujeitos.
\end{abstract}

\section{INTRODUÇÃo}

O estabelecimento da relação estruturafunção nas moléculas de proteínas tem sido uma problemática fundamental a elucidar. A hemoglobina graças ao amplo conhecimento das suas propriedades, tem se comportado como modelo para o estabelecimento desta relação, possibilitando um grande avanço no estudo das proteínas em geral (Briehl, 1963) .

A hemoglobina, hemoproteína transportadora de oxigênio do meio ambiente até os tecidos nos seres vivos, desempenha sua função em condições extremamente variáveis, dependendo das necessidades do organismo e do meio ambiente em que se desenvolve. Um estudo comparativo, considerando estes aspectos, nos possibilita a identificação dos mecanismos adaptativos a nível molecular, segundo os quais, os diferentes organismos adaptam-se às condições ambientais.

A propriedade fundamental da hemoglobina que consiste na oxigenação, acarreta na molécula de hemoproteína alterações estruturais profundas (Perutz et al., 1969), por exemplo: variação no equilíbrio de associação-dissociação das subunidades (Wyman, 1964). A sua saturação pelo gás é o resultado de interações homotrópicas e heterotrópicas, as primeiras foram descritas como mudanças conformacionais dentro de uma cadeia polipeptídica, originada pela interação entre os grupos heme, e as interações heterotrópicas por sua vez, às modificações nos sítios de união de um ligante por efeito de outros ligantes interações que envolvem mais de uma cadeia polipeptídica. Os protóns e fosfatơs orgânicos que afetam a curva de equilíbrio de oxigênio, correspondem às interações heterotrópicas (Brunori, 1975) . A presença simultânea destas interações, apresenta vantagem para a hemoglobina, visto que as vezes pode realizar o transporte de oxigênio em condições de metabolismo adversas.

A função da hemoglobina freqüentemente parece estar adaptada às necessidades metabólicas do animal e ao meio ambiente, incluindo mudanças intrínsicas da afinidade pelo oxigênio, mudanças no conteúdo de efeitos alostéricos e variação no cooperativismo de interaçãc pelas uniões sucessivas das moléculas de oxigênio (Riggs, 1976).

Em peixes, tem-se observado que existem várias maneiras de suprir as diferentes necessidades de oxigênio, entre estas, a presença de brânquias com diferente área efetiva de troca de oxigênio, variação do pulso cardíaco,

(*) - Instituto Nacional de Pesquisas da Amazônia, Manaus. (*) - Universidade Estadual de Campinas, SP. 
do número de glóbulos vermelhos, da concentração de hemoglobina, e a presença de uma hemoglobina especial, parecem constituir as mais importantes (Riggs, 1970). Em diferentes espécies de teleósteos, a quantidade de eritrócitos e a concentração de hemoglobina no sangue dependem da quantidade de oxigênio disponivel e da temperatura (Dube et al., 1973). Outra correlação achada em teleósteos marinhos foi que a área superficial das brân. quias por grama de peso, em peixes ativos, é duas vezes maior que em peixes lentos (Gray, 1954; Hughes, 1966; Hughes et al., 1970).

As propriedades funcionais da hemoglobina também parecem estar adaptadas às necessidacies respiratórias de cada espécie. Os fatores que regulam a afinidade pelo oxigênio bem como outras propriedades funcionais, podem estar relacionados com a disponibilidade de oxigênio dissolvido na água e atividade metabólica característica de cada peixe (Grigg, 1974) .

$\mathrm{Da}$ classe dos vertebrados, a hemoglobina de peixes parece ser mais heterogênea e diversificada, sugerindo-se que esta em termos de sua funçăo, esteja adaptada às condiçōes variáveis de oxigênio do meio ambiente ou a um sistema de interação individual na molécula para produzir um equilíbrio com o oxigênio específico no sangue (Grigg, 1974). existindo numerosas formas adaptativas da hemoglobina, relacionadas com os diversos parâmetros ambientais (Powers et al., 1972).

Tendo-se como objetivo verificar as adaptações da hemoglobina em relação a sua função fisiológica com a do meio ambiente, foram escolhidos dois peixes bastante característicos quanto ao seu tipo de respiração, atividade e meio ecológico, Osteoglossum bicirrhossum, aruanä, e Pterygoplicthys pardalis, acari-bodó, o primeiro caracteriza-se por ser um peixe bastante ativo, de respiração branquial, encontrado comumente em águas superficiais, pouco resistente a conteúdos baixos de oxigênio, e o segundo, um peixe lento de águas profundas, de respiração aérea facultativa, provido de uma grande resistência para permanecer em condições de baixa oxigenação. Estes peixes, encontrados em água doce de regiões tropicais, especificamente na Amazônia, possuem adaptações fisiológicas conhecidas, constituindo portanto, material biológico Interessante para estudar os diferentes mecanismos adaptativos que se desenvolvem nos diversos organismos, para satisfazer às suas necessidades metabólicas em relação ao seu meio ecológico. Estes estudos embora preliminares constituem o objetivo deste trabalho.

\section{MATERIAIS E MÉTODOS}

Exemplares das duas espécies, aruanã, (Fig. 1) e de acari-bodó, (Fig. 2), foram coletados no rio Solimões, região de Janauacá, no perícdo de novembro de 1976 a janeiro de 1977. Animais de pesos e tamanhos homogêneos foram sangrados por punção cardíaca, mediante seringa heparinizada com 5000 unidades de heparina por mililitro. As hemácias foram lavadas 3 a 4 vezes com solução de $\mathrm{NaCl} 1,7 \%$ contendo Tris $1 \mathrm{mM}$ pH 8,0 a $4^{\circ} \mathrm{C}$, por centrifugações sucessivas a $5000 \mathrm{rpm}$. Para obtenção do hemolisado das células foram lisadas com solução de Tris $1 \mathrm{mM}$ pH 8,0 na proporção de 2 a 3 volumes de sangue e após adicionado $\mathrm{NaCl} 1 \mathrm{M}$ até uma concentração final de $0,1 \mathrm{M}$, a solução de hemoglobina foi finalmente obtida após centrifugação a 15000 $\mathrm{rpm}$. Para a preparação da solução de hemoglobina desionisada, foi utilizado o método cromatográfico descrito por Noble et al. (1976).

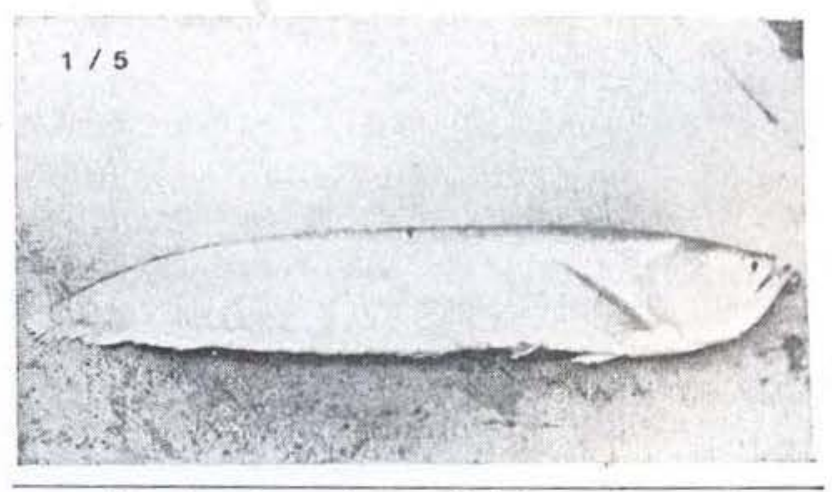

Fig. 1-Osteoglossum bicirrhossum, aruanā

Na identificação dos componentes de hemoglobina, por eletroforese em gel de poliacrilamida, foi utilizada a técnica descrita por Ornstein (1964) e Davis (1964) usando gel de 
poliacrilamida $7,5 \%$ pH 8,9. Na separação dos componentes identificados por eletroforese. utilizou-se coluna de troca iônica, DEAE-Sephade A-50 e eluída a amostra em um gradiente linear de $\mathrm{pH}$ utilizando-se tampão Tris- $\mathrm{Cl}$ 0,05 $\mathrm{M} \mathrm{pH} 9,0$ e Tris-Acetato $0,05 \mathrm{M} \mathrm{pH} \mathrm{6,0.}$

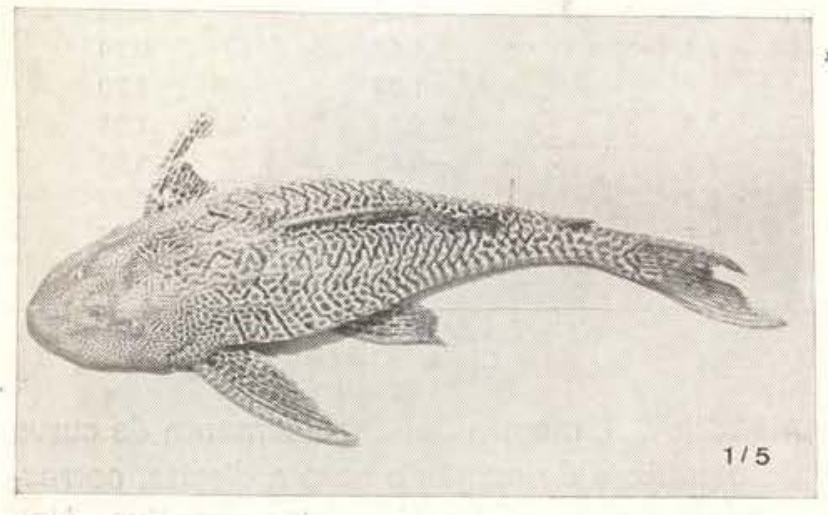

Fig. 2 - Pterygoplicthys pardalis, acari-bodó

C equilíbrio de hemoglobina com oxigênio foi medido pelo método de Riggs et al. (1976), utilizando-se soluções de hemoglobina deionizada no $\mathrm{pH}$ adequado para cada análise. A desoxigenação da hemoglobina foi verificada espectrofotometricamente e as curvas espectrais obtidas após oxigenação gradual.

$\mathrm{O}$ efeito Bohr ou efeito do $\mathrm{pH}$ na curva de equilíbrio de oxigênio foi analisado utilizandose tampões Tris ou Bis-Tris com força iônica $\mathrm{I}=0,05$, entre valores de $\mathrm{pH} 6,0$ a 9,0 .

A análise de fosfato como efetor alostérico na curva de equilíbrio de oxigênio, foi estudado através de determinações das curvas de equilíbrio da hemoglobina com oxigênio na presença de ATP $10^{-3} \mathrm{M}$, em tampão Tris ou Bis-Tris, $\mathrm{I}=0,05$ entre valores de $\mathrm{pH} 6,0$ a 9,0 .

\section{Resultados}

IDENTIFICAÇÄO DOS COMPONENTES DE HEMOGLOBINA POR ELETROFORESE EM POLIACRILAMIDA

Submetida à eletroforese em poliacrilamida, a hemoglobina de aruanã apresentou-se em numerosas análises, como um componente único, com pequena migração anódica, enquan- to que a hemoglobina de acari-bodó apresentou quatro componentes, com mobilidades eletroforéticas relativas diferentes, que foram denominadas Hbl, Hbll, Hblll e HbIV. Em função das mobilidades anódicas decrescentes, Figura 3 , por eletroforese realizadas individualmente, não revelaram variações apreciáveis em seus perfís, sugerindo-se a inexistência, nas condições descritas, de polimorfismo nas espécies examinadas.

\section{SEPARAÇÃO DOS COMPONENTES DE} HEMOGLOBINA POR CROMATOGRAFIA DE TROCA IỐNICA

Do hemolisado de acari-bodó deionisado, eluído em gradiente linear de $\mathrm{pH}$ com Tris- $\mathrm{Cl}$ $0,05 \mathrm{M} \mathrm{pH} 9,0$ e Tris-Acetato $0,05 \mathrm{M}$ pH 6,0, numa coluna de troca iônica DEAE-Sephadex A-50, foram obtidas quatro frações, a primeira eluída a $\mathrm{pH} 9,0$ correspondente ao primeiro componente eletroforético, $\mathrm{Hbl}$ e as outras três frações menores, Hbll, Hblll e HbIV, eluídas em $\mathrm{pH}$ decrescente, Figura 4.

\section{EFEITO BOHR NA HEMOGLOBINA DE ARUANÃ}

$\mathrm{O}$ efeito Bohr ou efeito do $\mathrm{pH}$ nas curvas de equilíbrio de oxigênio de aruanã, expressos em forma logarítmica, Figura 5, Tabela I, mostram a grande dependência do $\mathrm{pH}$ na curva de saturação de hemoglobina com oxigênio, obtendo-se a $\mathrm{pH}$ baixo 5,5 a 6,5 uma saturação

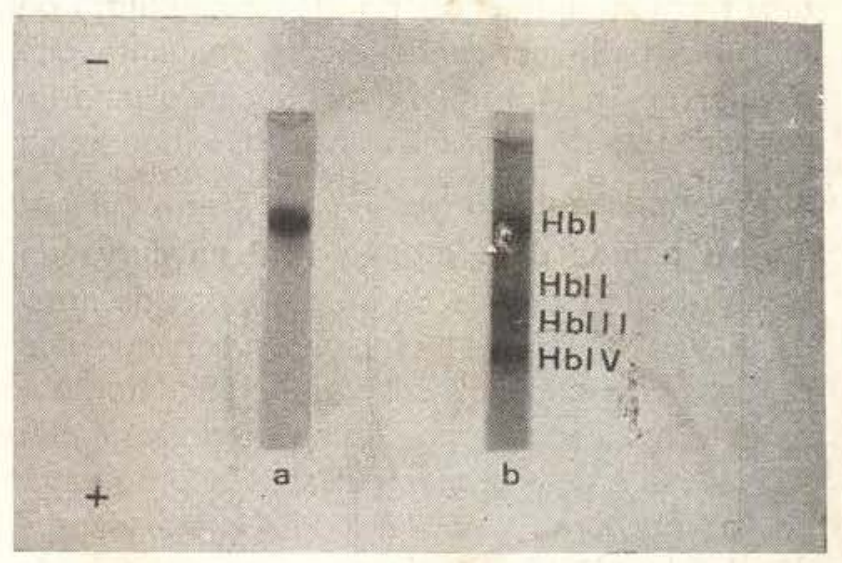

Fig. 3 -Componentes de hemoglobina por eletroforese em poliacrilamida: a) em aruanã; b) em acari-bodó. 


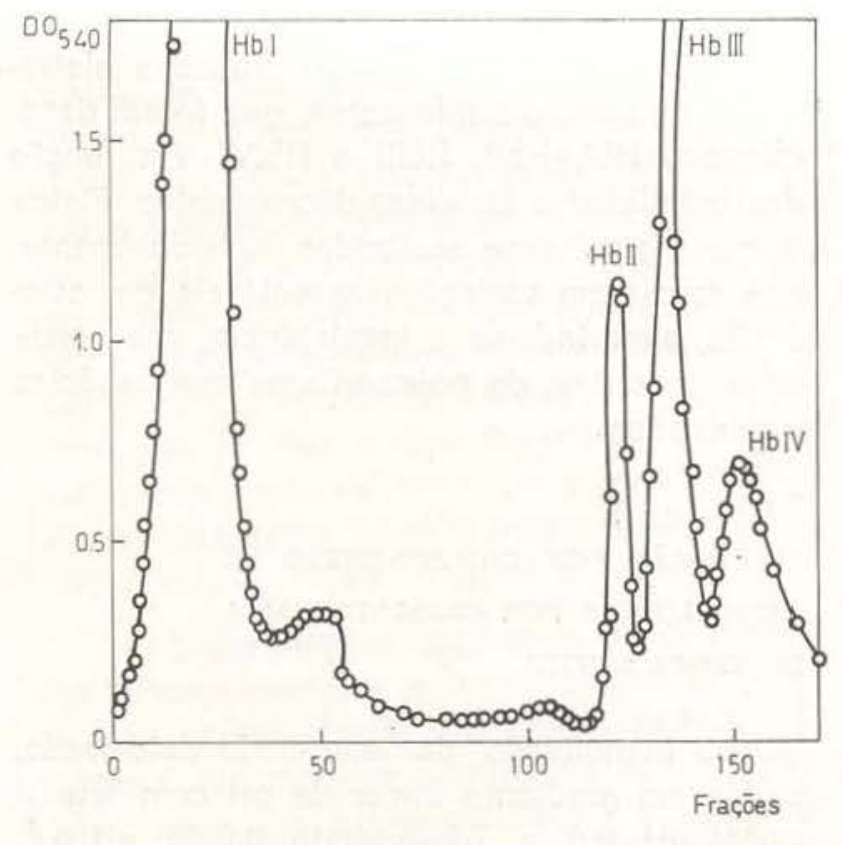

Fig. 4 - Separação dos componentes de hemoglobina de acari-bodó, por cromatografia de troca iônica, DEAE-SEPHADEX-A50 em gradiente de $\mathrm{pH}$.

com o ligante menor de $50 \%$ indicando uma marcada resistência da molécula a ser saturada com oxigênio (efeito Root), e uma cooperatividade negativa entre os sítios heme nestes $\mathrm{pH}$. No intervalo de $\mathrm{pH} 6,5$ a 7,0 observou-se uma maior sensibilidade de hemoglobina pelo oxigênio, pois com uma pequena mudança de $\mathrm{pH}$, obteve-se grande variação da afinidade da mesma pelo gás e o valor de " $n$ " neste intervalo de $\mathrm{pH}$ tornou-se maior que 1 , indicando uma interação favorável, entre os sítios. Já em intervalo de pH superior entre 7,6 e 9,2, uma pequena influência do $\mathrm{pH}$ no equilíbrio de oxigênio pode ser evidenciada, enquanto que $o$ valor de " $n$ " foi igual a 1, indicando uma independência de interação entre os sítios heme nesta faixa de $\mathrm{pH}$.

No estudo do efeito de ATP na curva de equilíbrio de oxigênio de hemoglobina de aruanã, Figura 6, Tabela II, foi observado que nos $\mathrm{pH}$ descritos entre 5,8 a 7,2 , há menos de $50 \%$ de saturação dos grupos heme na presença de ATP $10^{-3} \mathrm{M}$ e uma grande variabilidade do valor de " $n$ " em torno de 0,5 a 2,0. A maior dependência do $\mathrm{pH}$ observada entre os valores de 6,5 a 7,0 na ausencia de ATP comparada aos valores obtidos na sua presença em torno de
TABELA I - Efeito do $\mathrm{pH}$ no equilibrio de oxigênio em função de $\log P_{50}$ e na cooperatividade, valor de " $n$ " na hemoglobina de aruanã

\begin{tabular}{crc}
\hline $\mathbf{p H}$ & $\log \mathbf{P}_{\mathbf{5 0}}$ & $\mathbf{n}$ \\
\hline 5,7 & 1,96 & 0,55 \\
6,1 & 1,92 & 0,48 \\
6,4 & 1,60 & 0,30 \\
6,7 & 1,04 & 1,20 \\
6,9 & 0,64 & 1,83 \\
7,6 & $-0,16$ & 1,00 \\
8,2 & $-0,24$ & 0,87 \\
8,7 & $-0,32$ & 0,85 \\
9,2 & $-0,36$ & 1,09 \\
\hline
\end{tabular}

$\mathrm{pH} 7,2$ a 7,5 , mostra um deslocamento da curva de equilibrio de oxigênio para a direita, correspondente a um efeito alostérico negativo. Nos $\mathrm{pH} 7,6$ a 9,0, visualizamos um efeito menor do ATP $10^{-3} \mathrm{M}$ e um valor inalterado de " $\mathrm{n}$ ".

$\mathrm{A}$ afinidade de hemoglobina de aruană, $\mathrm{Fi}$ gura 7 , confirma o grande efeito Bohr com um valor de $\Delta \log \mathrm{P}_{50} / \Delta \mathrm{pH}=-1.4$, pronunciada dependência da afinidade de hemoglobina pelos valores de $\mathrm{pH}$. Esta curva, na presença de ATP $10^{-3} \mathrm{M}$, revela valores mais altos de log $P_{50}$, indicando um efeito alostérico negativo, máximo a pH 7,0. Os efeitos menores sāo encontrados a pH 8,7 e 5,7 .

Resultados comparativos dr, equilibrio de oxigênio na hemoglobina na presença e ausência de ATP, Tabela II, mostram nitidamente a dependência em função do $\mathrm{pH}$ e a grande variabilidade de cooperativismo existente entre os sítios de união do oxigênio nos diferentes $\mathrm{pH}$.

\section{EFEITO BOHR EM HEMOGLOBINA DE ACARI-BODÓ}

A análise do equilíbrio de oxigênio em função do $\mathrm{pH}$ no hemolisado total de hemoglobina da outra espécie, acari-bodó, Figura 8, Tabela III, revelou pequena influência do $\mathrm{pH}$. com uma variação máxima de 0,2 unidades na afinidade da hemoglobina pelo oxigênio e um valor de " $n$ " próximo de 1 indicando a independência do $\mathrm{pH}$ no grau de interação entre os grupos heme. 


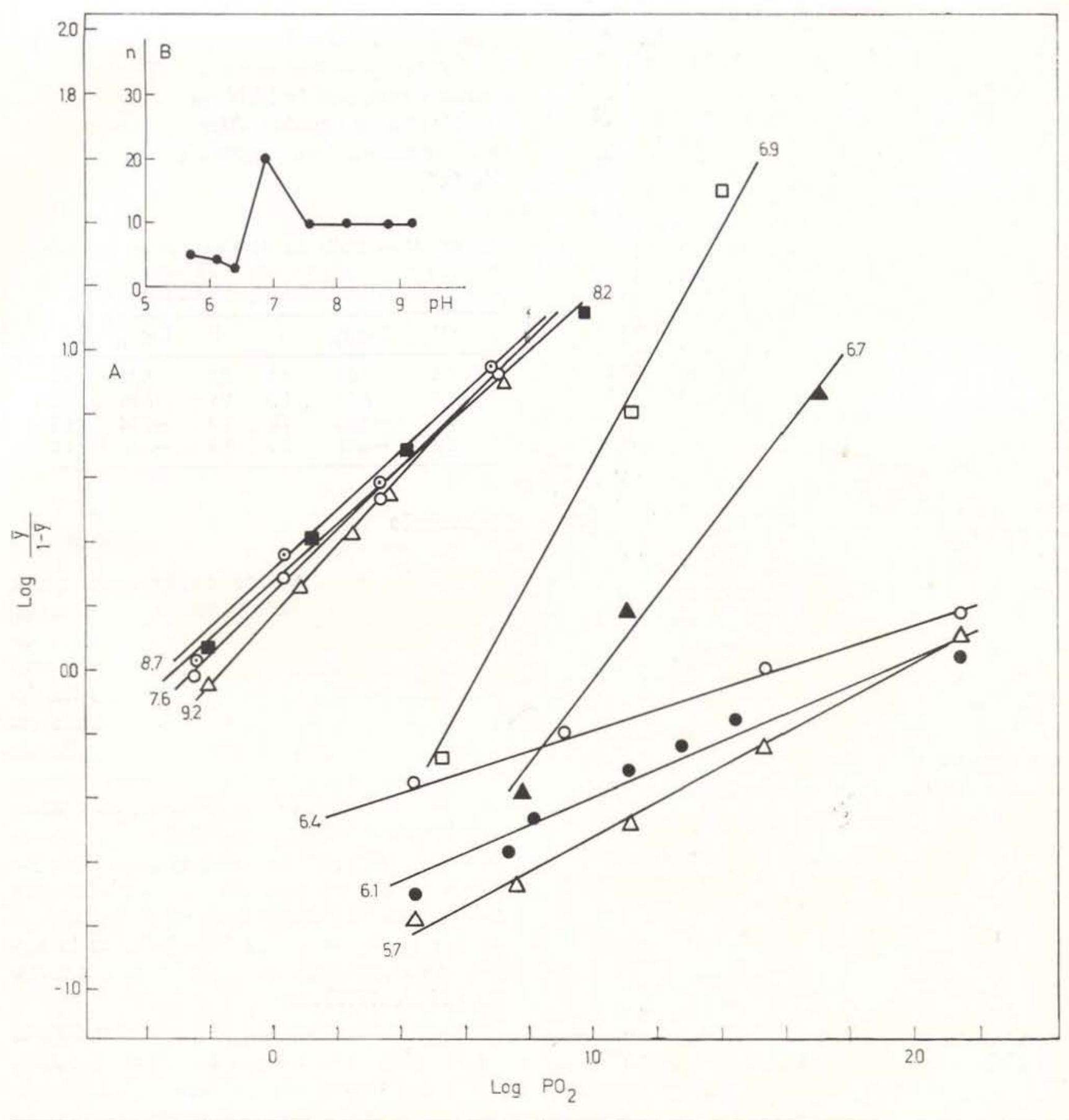

Fig. 5 - Efeito do $\mathrm{pH}$, a) na curva de equilíbrio de hemoglobina com oxigênio; b) na cooperatividade de oxigênio com hemoglobina, de aruană.

O exame da curva de oxigênio dos componentes isolados de hemoglobina de acari-bodó, nos levou a diferentes resultados, assim o componente $\mathrm{Hbl}$, o de menor mobilidade anódica, é o primeiro a ser eluido da coluna de DEAE-Sephadex A-50, mostrou, na curva de equilíbrio de oxigênio, Figura 9, e Tabela IV, um pequeno efeito Bohr reverso, com um valor máximo de $\Delta \log \mathrm{P}_{50} / \Delta \mathrm{pH}=0,22$ a valolores de $\mathrm{pH} 7,4$ a 8,3 , indicando uma maior afinidade de oxigênio por hemoglobina a $\mathrm{pH}$ baixo. Na presença de ATP $10^{-3} \mathrm{M}$, um efeito oposto pode ser observado, obtendo-se um efeito Bohr normal de valor $-0,18$ a $\mathrm{pH} 7,4 \mathrm{e}$ 
TABELA II - Efeito do pH e ATP no equilibrio de hemoglobina com oxigênio em funçăo de $\log \mathrm{P}_{50}$ na cooperatividade de oxigênio com hemoglobina, valor de "n", de aruanã

ATP $10^{-3} \mathrm{M}$

\begin{tabular}{crrrrr}
\hline $\mathbf{p H}$ & $\log \mathbf{P}_{\mathbf{5 0}}$ & $\mathbf{n}$ & $\mathbf{p H}$ & $\log \mathbf{P}_{\mathbf{5 0}}$ & $\mathbf{n}$ \\
\hline 5,7 & 1,96 & 0,55 & 5,8 & 1,96 & 0,45 \\
6,1 & 1,92 & 0,48 & 6,1 & 2,04 & 0,44 \\
6,4 & 1,60 & 0,30 & 6,9 & 1,52 & 1,00 \\
6,7 & 1,04 & 1,20 & 7,2 & 1,08 & 1,12 \\
6,9 & 0,64 & 1,83 & 7,4 & 0,56 & 2,00 \\
7,6 & $-0,16$ & 1,00 & 7,5 & 0,18 & 1,28 \\
8,2 & $-0,24$ & 0,87 & 7,6 & 0,04 & 1,30 \\
8,7 & $-0,32$ & 0,85 & 8,7 & $-0,28$ & 1,20 \\
9,2 & $-0,36$ & 1,09 & 9,0 & $-0,16$ & 1,00 \\
\hline
\end{tabular}

8,3. Este efeito tende a desaparecer a pH acima de 8,0 . Os valores obtidos de afinidade de oxigênio no componente $\mathrm{Hbl}$, em função de $\log \mathrm{P}_{\mathrm{so}}$ indicam uma alta afinidade a $\mathrm{pH}$ baixo, estes valores se invertem na presença de ATP $10^{-3} \mathrm{M}$. Os valores de " $n$ " mostram-se maior que 1 , indicando uma pequena interação cooperativa entre os grupos heme. O ATP neste caso não apresentou nenhum efeito evidente.

No componente HblV, que corresponde ao componente de maior migração eletroforética anódica, a curva de equilíbrio de oxigênio,

TABELA III - Efeito do pH no equilibrio de oxigênio e na cooperatividade de oxigênio nos componentes totais de hemoglobina de acari-bodó

\begin{tabular}{crc}
\hline pH & Log $\mathbf{P}_{50}$ & $\mathbf{n}$ \\
\hline 6,6 & 0,04 & 1,3 \\
7,0 & $-0,12$ & 1,3 \\
7,8 & $-0,20$ & 1,3 \\
8,3 & $-0,16$ & 1,0 \\
\hline
\end{tabular}

TABELA IV - Efeito do pH e de ATP no equilíbrio de oxigênio e na cooperatividade dé oxigênio do componente $\mathrm{Hbl}$ de acari-bodó

ATP $10^{-3} \mathrm{M}$

\begin{tabular}{cccccc}
\hline $\mathbf{p H}$ & $\log \mathbf{P}_{\mathbf{5 0}}$ & $\mathbf{n}$ & $\mathbf{p H}$ & $\log \mathbf{P}_{\mathbf{5 0}}$ & $\mathbf{n}$ \\
\hline 6,7 & 0,15 & 1,87 & 6,7 & 0,70 & 1,4 \\
7,4 & 0,22 & 1,40 & 7,4 & 0,51 & 1,6 \\
7,8 & 0,25 & 1,54 & 7,8 & 0,40 & 1,5 \\
8,3 & 0,42 & 1,56 & 8,3 & 0,34 & 1,6 \\
8,8 & 0,39 & 1,40 & 8,8 & 0,36 & 1,5 \\
\hline
\end{tabular}

Figura 9, Tabela V, mostrou um efeito Bohr considerado normal, com um valor de $\Delta \log$ $\mathrm{P}_{\mathrm{so}} / \Delta \mathrm{pH}=-0,66$ entre os $\mathrm{pH} 6,2$ e 8,2 . O mesmo componente HbIV na presença de ATP $10^{-3} \mathrm{M}$ não apresentou diferenças apreciáveis, observando-se uma pequena variação no valor de " $n$ ".

TABELA V - Efeito do pH e de ATP no equilibrio de oxigênio e na cooperatividade de oxigênio do componente HblV de hemoglobina de acari-bodó

\begin{tabular}{rrrrrr}
\hline $\mathbf{p H}$ & $\log \mathbf{P}_{\text {50 }}$ & $\mathbf{n}$ & $\mathbf{p H}$ & $\log \mathbf{P}_{\mathbf{5 0}}$ & $\mathbf{n}$ \\
\hline 6,2 & 1,20 & 1,3 & 6,2 & 1,00 & 1,0 \\
6,5 & 0,50 & 1,3 & 7,1 & 0,00 & 1,7 \\
7,5 & $-0,08$ & 1,9 & 7,6 & $-0,04$ & 1,5 \\
8,2 & $-0,11$ & 1,1 & 8,4 & $-0,26$ & 1,2 \\
\hline
\end{tabular}

\section{Discussão}

Evidências anteriores estabelecem que a multiplicidade de componentes observados, por diferentes métodos nas hemoglobinas de diferentes espécies, podem estar correlacionados tanto como as suas propriedades fisiológicas, como com as variaçōes ambientais em que vivem os animais. Assim, peixes vivendo em regiōes de grande variabilidade térmica, apresentam hemoglobinas para suportar estas condições e conseqüentemente, temos populações de maior polimorfismo, enquanto que padrões de hemoglobina menos complexos com um ou dois componentes são encontrados em peixes marinhos de profundidade, meio ambiente de pequena variação térmica (Bonaventura et al., 1975).

Estas assertivas, contudo, parecem difíceis de ser aplicadas ao nosso caso, pois o aruanã, peixe que vive em águas superficiais sujeitas às variações de temperatura, apresenta uma única hemoglobina de acordo com critérios eletroforéticos e cromatográficos. O acari-bodó, animal que vive em águas de maior profundidade, de menor variação de temperatura, apresenta quatro componentes eletroforéticos. sendo o de maior proporção, catódico, e os três outros componentes, anódicos. Evidentemente, de acordo com nossos resultados, outros fatores não só a temperatura, estão participando na multiplicidade dos componentes encontrados no acari-bodó. 


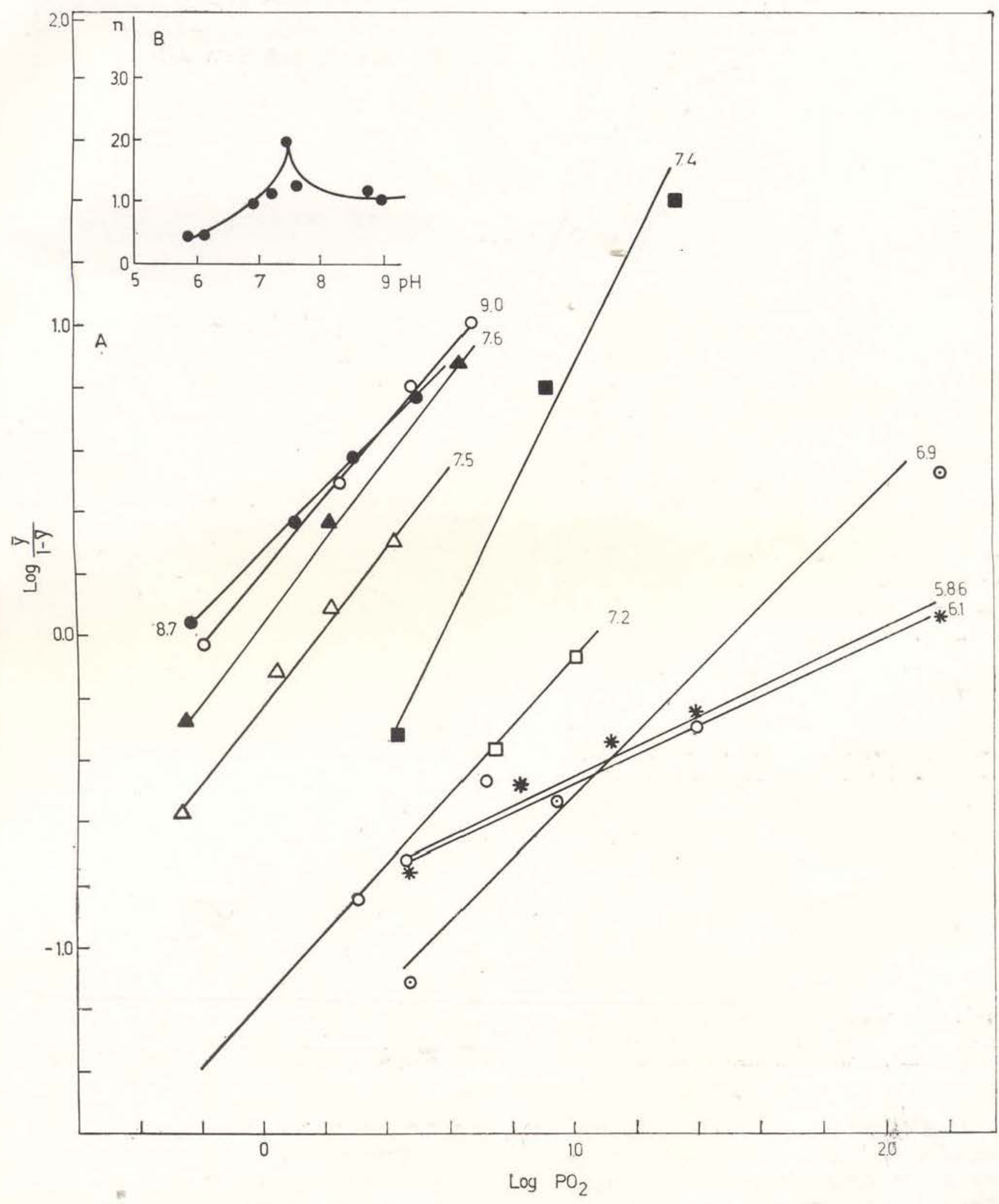

Fig. 6 - Efeito do $\mathrm{pH}$ e ATP $10^{-3} \mathrm{M}$. a) na curva de equilibrio de hemoglobina com oxigênio; b) na cooperatividade de oxigênio com hemoglobina, em aruanå.

Estudos... 


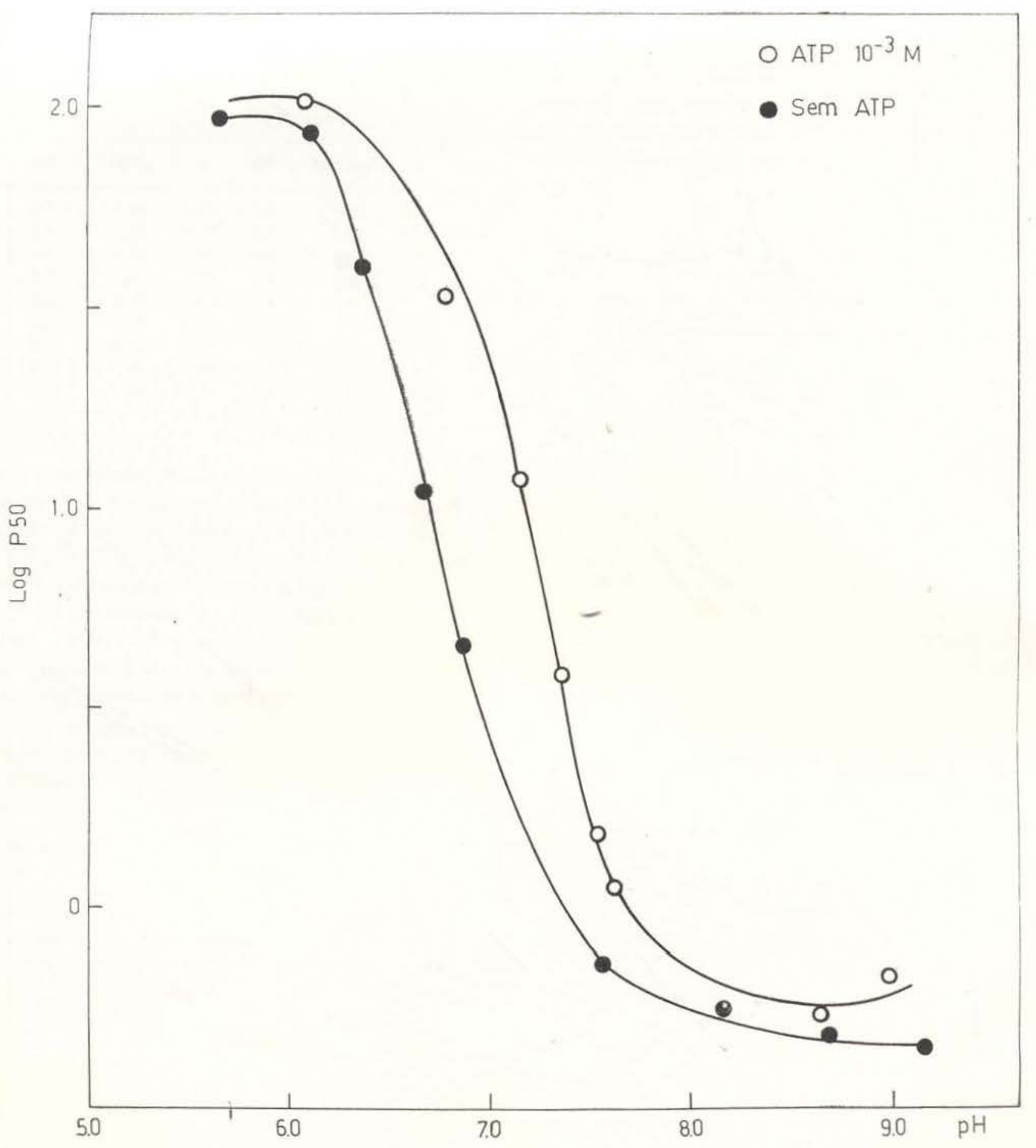

Fig. 7 - Afinidade de hemoglobina de aruanã em termos de $\log \mathrm{P}_{50}$ e pH, na presença e ausência de ATP.

Mais do que a multiplicidade de componentes, a afinidade destes pelo oxigênio em peixes, parece estar relacionada com as condições ambientais, assim, os valores de $P_{50}$ são proporcionais à quantidade de oxigênio presen. te no meio, encontrando-se geralmente valores baixos para $50 \%$ de saturação de hemoglobina, naqueles que vivem em águas pouco oxigenadas, indicando sua alta afinidade pelo ligante. Nossos resultados e outros anteriores, corroboram estas afirmativas já que a afinidade da hemoglobina pelo oxigênio encontrada no acari-bodó é alta e conseqüentemente os valores $\mathrm{P}_{50}$ em pH em torno de 7,0 são baixos da ordem 
de $0,69 \mathrm{~mm}$ de $\mathrm{Hg}$. O aruanã, por outro lado, sendo peixe que vive em águas superficiais muito oxigenadas, os valores de $\mathrm{P}_{50}$ encontrados nas mesmas condições são mais altos da ordem de $4,36 \mathrm{~mm}$ de $\mathrm{Hg}$, indicando uma menor afinidade da hemoglobina pelo oxigênio.

O efeito Root (Root, 1931), isto é, a insaturação da hemoglobina pelo oxigênio em $\mathrm{pH}$

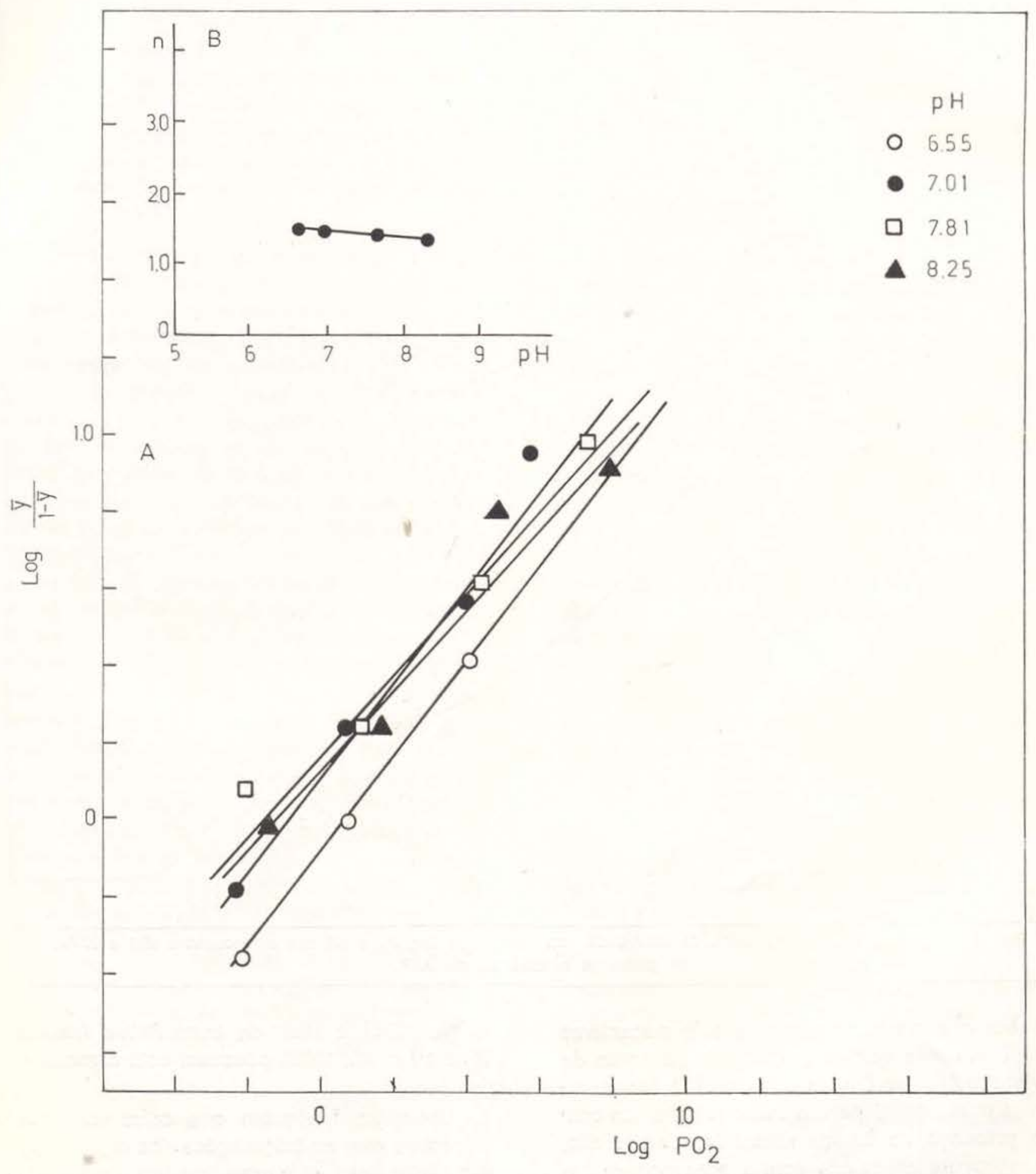

Fig. 8 - Efeito do pH. a) na curva de equilibrio de hemoglobina com oxigênio; b) na cooperatividade de oxigênio com hemoglobina, de acari-bodó. 


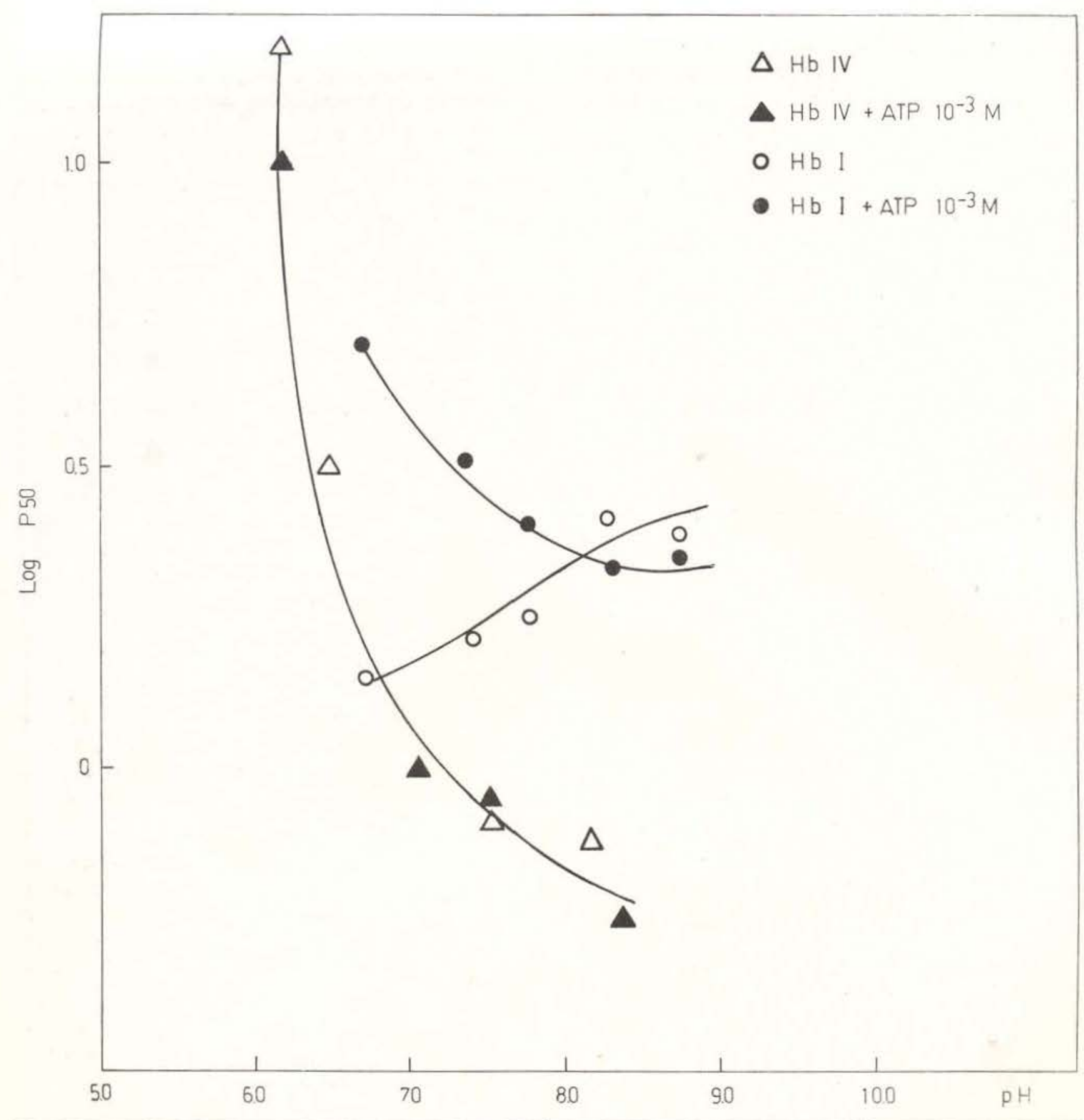

Fig. 9 - Afinidade de hemoglobina de acari-bodó, em termos de Log $\mathrm{P}_{50}$ e pH nos componentes Hbl e HbIV, na presença e ausência de ATP.

baixo, observado no aruanã e cujo mecanismo não tem sido bem compreendido do ponto de vista molecular (Noble et al., 1970) (Bonaventura et al., 1976) parece estar relacionado com a presença de bexiga natatória. Este efeito, geralmente associado com a presença de bexiga natatória nas hemoglobinas, amplamente estudados em carpa Ciprinus carpio (Noble et al., 1970) e HblV de truta Salmo iredeus (Brunori et al., 1973) possuem esta característica.

Um outro fenômeno que sofre variações de acordo com as exigenações dos organismos é o efeito Bohr, cuja grandeza tem se mostrado em muitas espécies, ser diretamente proporcional ao grau de atividade dos indivíduos 
(Riggs. 1970). Nossos dados com relação ao hemolisado total atestam esta correlação. As$\operatorname{sim}$, o aruanã, peixe de grande atividade motora, possui alto efeito Bohr com um valor de $\Delta \log \mathrm{P}_{50} / \Delta \mathrm{pH}$ de -1.44 enquanto que no acari-bodó, lento, este valor diminui para $-0,66$. Evidentemente, no caso do aruanã, que apresenta uma única hemoglobina o valor de efeito Bohr encontrado deverá corresponder ao do hemolisado, no acari-bodó. Entretanto, graças aos seus quatro componentes, este efeito representa no hemolisado, na verdade, uma média dos efeitos de cada componente separado

De acordo com os autores Riggs (1976); Brunori (1975); Powers (1974) os vários componentes da hemoglobina no hemolisado, corresponde a proteinas com propriedades funcionais diferentes, fisiologicamente econômicas para o organismo. Assim, o acari-bodó se mostrou em nossos resultados como construído de duas familias de hemoglobina, uma que representa cerca de $50 \%$ do hemolisado total com propriedades funcionais diferentes das 3 outras cujas propriedades se apresentam muito semelhantes entre si, mas profundamente diferentes do primeiro componente. De fato, o componente denominado $\mathrm{Hbl}$ apresentou pequeno efeito Bohr reverso da ordem de $+0,22$ que tende à normalidade na presença de efetores heterotrópicos. ATP com um valor de $-0,18$, enquanto que HbIV apresenta efeito Bohr chamado normal com um valor de $-0,66$, isto é, semelhante a da hemoglobina humana e nenhum efeito de ATP. Baseado nisto, poderíamos concluir que o acari-bodó apresenta hemoglobina $\mathrm{Hbl}$ capaz de suprir uma oxigenação adequada em caso de exercícios intensos onde os niveis de ácido lático são elevados.

Se podemos comparar nossos resultados com os encontrados em hemoglobinas de truta e carpa, alguma correlação entre estrutura e função pode ser alcançada. Assim, a truta apresenta quatro hemoglobinas, com dois componentes funcionalmente diferentes $\mathrm{Hbl}$, isenta de efeito Bohr e HbIV com efeito Bohr elevado além de efeito Root (Brunori, 1975) cujas cadeias polipeptidicas $\propto$ e $\beta$ dieferem profundamente em sua estrutura primária (Barra et al., 1973). A carpa com vários com- ponentes, funcionalmente semelhantes entre si (Noble et al., 1970; Gilln et al., 1972) as cadeias polipeptidicas $\propto$ são idênticas diferindo exclusivamente na cadeia $\beta$ (Hilse et al. 1968). Do exposto pode-se inferir que o acaribodó, com hemoglobinas funcionalmente diferentes, deve diferir apreciavelmente em sua estrutura primária.

$\mathrm{O}$ aruană com um único componente a hemoglobina está preparada para exercer diferentes funções, de acordo com as necessidades fisiológicas e ambientais do peixe, desta forma, tanto a estrutura primária, como mudanças conformacionais ocorridas pelo ligante, responderiam pela multiplicidade de funçōes da hemogiobina. Na verdade o coeficiente de Hill em diferentes $\mathrm{pH}$ dāo idéias mais ou menos reais a respeito de mudanças conformacionais induzidas pelos efeitos alostéricos. Assim os valores de " $n$ " encontrados para o arua:nā, variáveis entre 0,9 a 0,18 a $\mathrm{pH} 6,0$ a 7,0 são coincidentes com o alto efeito Bohr encoritrado nestes peixes. Já no hemolisado total de acari-bodó onde os valores de " $n$ " são praticamente constantes o efeito Bohr é menor. De acordo com nossos resultados as hemoglobinas de acari-bodó que parecem ser funcionalmente diferentes, os valores de " $n$ " nâo variam de maneira considerável, indicando que as interações homotrópicas não são tão importantes como as heterotrópicas. Por outro lado, uma variação ponderável no valor de " $n$ " parece ser encontrado em hemoglobinas funcionalmente semelhantes entre elas ou naquele que se apresenta com um único componente como o caso de aruanā. O efeito Root encontrado tanto no aruanã como na carpa, estaria de alguma forma associado a esta variaçäo nos valores de " $n$ ".

Finalmente, podemos concluir com os estudos destas duas espécies de peixes do rio Amazonas que muitas regras gerais estabelecidas para peixes de outras regiōes, são confirmadas, outras contudo. como o caso da multiplicidade de componentes em relação a temperatura em que vive o animal, não o foram. Na verdade, o rio Amazonas apresenta-se com caracteristicas próprias, impondo um ambiente de grande variabilidade à vida dos peixes e estes, por sua vez, devem apresentar 
características vitais das mais diversas para suportar aquela variação e isto evidentemente se estende à hemoglobina. No futuro, outras espécies de peixes do rio Amazonas estarão sendo estudadas com pormenores, sempre com o objetivo de conhecer melhor o ponto crítico da natureza e a relação que existe entre a estrutura das biomoléculas e suas funções.

\section{AGRADECIMENTOS}

Ao Dr. Warwick Estevam Kerr, Diretor do Instituto Nacional de Pesquisas da Amazônia pelo grande incentivo dado à pesquisa na Amazônia e que tornou possivel a realização deste trabalho, ao Dr. Austen Riggs, chefe da "Alpha Helix IV Expedition, 1976", e pesquisadores participantes, pelo estímulo dado na pesquisa da hemoglobina. Ao Dr. H. A. Britski do Museu de Zoologia da Universidade de São Paulo, pela classificaçăo taxonômica do material biológico.

\section{SUMMARY}

Two species of fish hemoglobin from Amazon river were analized in order to establish the relationship between their molecular and physiological properties as well as their interactions with other molecules and ions. The species were different regarding respiration, activity and habitat. The polyacrylamide eletrophoresis pattern of aruană hemoglobin showed only one component and pronounced Bohr and Root effect and the Hill coefficient showed significant variation with the $\mathrm{pH}$ in the presence or not of $10^{-3} \mathrm{M}$ ATP. The total hemolisate of acari-bodó presented not Bohr effect nevertheless the four isolated components showed different functional properties thus the $\mathrm{Hbl}$ component, showed small reverse Bohr effect that becomes normal in the presence of $10^{-3} \mathrm{M}$ ATP at low $\mathrm{pH}$. The other three components presented normal Bohr effect and ATP does not change this effect. These results cleary show the capacity of molecular adaptation of fish hemoglobin to adapt to both the metabolic requirements and environment variations of the animals.

\section{BIBLIOGRAFIA}

Barra, D.: Bossa, F.: Bonaventura, J. \& Brunori, M. 1973 - Hemoglobin components from trout (Salmo iredeus) : determination of the carboxy and amino terminal sequences and their functional implications. FEBS Letters, 35 : 151-154.
Bonaventura, C. Sullivan, B. \& Bonaventura, J.

1976 - Spot hemoglobin studies on the Root effect hemoglobin of a marine teleosts. J. Biol. Chem., 251: 1871-1876.

Bonaventura, J.: Bonaventura, C. \& Sullivan, B. 1975 - Hemoglobins and hemocyanins: Comparative aspects of structure and function. J. Exp, Zool., 194: 155-174.

BRIEHL, R.

1963 - The relation between the oxygen equilibrium and aggregation of subunits in lamprey hemoglobin. J. Biol. Chem., 238: 2361-2666.

BRUNORI, M,

1975 - Mo!ecular adaptation to Physiological requirements: The hemoglobins Systems of trout, In: current topics in cellular regulation, 9: 1-38.

BrUNori, M.; Bonaventura, J.; Giardina, B.:

Bossa, F. \& ANTONINI, E.

1973 - Hemoglobins from trout: Structural and functional properties. Molec. Cell. Biochem., 1: 189-196.

Davis, B. J.

1964 - Disc electrophoresis II. Method and applications to human serum proteins. Ann. N.Y. Acad. Sci., 121: 404-427.

Dube, S. C. \& Mushi, J. S,

1973 - A quantitative study of the erythrocytes and hemoglobin in the blood of an Air-breathing fish. Anabas testudineus in relation to its body size. Folia Haematol., Leipzig, 100: 436-446.

Gillen, R. G. \& Riggs, A.

1972 - Structure and function of the hemog'obin of the carp, Cyprinus carpio. J. Biol. Chem., 247: 6039-6046.

Gray, E. I.

1954 - Comparative study of the gill area of marine fishes. Biol. Bull., 107: 219-225.

GrigG, G. C.

1974 - Respiration function of blood in fishes. Chem. Zool., 8: 331-368.

HiLse, K. \& BRAUnitzer, G.

1968 - Die aminosäuresequenz der der ketten der beiden kauptkomponenten des karpfenhänmoglobins. Hoppe-Seyler's Z Physiol. Chem., 349.: 433-450.

HUGHES, G. M.

1966 - The dimensions of fish gills in relation to their function. J. Exp. Biol., 45: 177-195. 
Hughes, G. M. \& Morgan, M. apud Johansen, Kjel! 1970 - Comparative Physiology: Gas exchange and circulation in fishes. Ann. Rev. Physiol. 33: 569-612, 1971.

Noble, B. \& Riggs, A.

1976 - In: Alpha Helix IV, Expedition, "Comunica. ção pessoal".

Noble, R. W.: Parkurst, L. \& Gibson, Q. H. 1970 - The effect of $\mathrm{pH}$ on the reactions of oxygen and carbon monoxide wite the hemoglobin of the carp, Cyprinus carpis. J. Biol. Chem., 245 : 6628-6633.

ORNSTEIN, L.

1964 - Disc electrophoresis I. Background and theory. Ann. N.Y. Acad. Sci., 121: 321-349.

Perutz M. F.; Mutrhead, H.; Mazzarella, L.; Crowther, R. A.; Greer, J. \& Kilmartin, J. V.

1969 - Identification of residues responsible for the alkaline Bohr effect in hemoglobin Nature, 222: 1240-1243.

PoWERS, D. A .

1974 - Structure function and molecular ecology of fish hemog'obins. Ann. N.Y. Acad. Sci., 241: 472-490.
Powers, D. A. \& Edmunson, A, B.

1972 - Multipes hemoglobins of Catastomid fish. 1. Isolation and Characterization of the isohemoglobins from Catostomus clarkii. J. Biol. Chem., 247 : 6686-6693.

RigGs, A.

1970 - Properties of fish hemoglobins. In: Fish Physiology. (Hoar W.S. \& Randall D.J.). New York, Acad. Press.. 4: 209-251.

1976 - Factors in the evolutions of hemoglobin function. Fedn. Proc., 35: 2115-2118.

Riggs, A. \& Noble, B.

1976 - In: Alpha Helix IV, Expedition, "comunicação pessoal".

Root, R. W.

1931 - The respiratory function of the blood of marine fishes. Biol. Bull., 61: 427-456.

WYMAN, J.

1964 - Linked functions and reciprocal effects in hemoglobin. A second look. Adv. Protein Chem., 19: 223-286.

(Aceito para publiçąão em 13/03/78) 